\title{
Influence of Sputtering Conditions for Co/Pd Multilayer on its Magnetic Properties and Crystalline Microstructure
}

\author{
Junichi Sayama", Jun Kawaji", Mutsumi Tanaka*, Toru Asahi" ${ }^{* *}$, Satoshi Matsunuma ${ }^{* * *}$, and Tetsuya Osaka ${ }^{*, * *}$ \\ ${ }^{*}$ School of Science and Engineering, Waseda University, 3-4-1 Ohkubo, Shinjuku-ku, Tokyo 169-8555, Japan \\ ${ }^{* *}$ Advanced Research Institute for Science and Engineering, Waseda University, 3-4-1 Ohkubo, Shinjuku-ku, Tokyo 169-8555, Japan \\ ${ }^{* * *}$ Development and Technology Division, Hitachi Maxell, Ltd., 6-20-1 Kinunodai, Yawaramura, Ibaraki 300-2496, Japan
}

The effects of substrate temperatures during deposition and $\mathrm{N}_{2}$ gas addition to Ar sputtering gas on the magnetic properties and crystalline microstructure of $\mathrm{Co} / \mathrm{Pd}$ multilayered films were investigated. The $\mathrm{Co} / \mathrm{Pd}$ multilayered film deposited at a substrate temperature of $230{ }^{\circ} \mathrm{C}$ with $\mathrm{Ar}$ containing $\mathrm{N}_{2}$ of $0.06 \mathrm{mT}$ Torr exhibited weaker intergranular exchange coupling than that deposited at room temperature without $\mathrm{N}_{2}$ addition. Plan-view TEM observation revealed that the crystal grains in the $\mathrm{Co} / \mathrm{Pd}$ multilayered film, which was subjected to the heating process with $\mathrm{N}_{2}$ additive gas, were physically isolated by vacant grain boundaries, resulting in the decrease in intergranular exchange coupling.

Key words: $\mathrm{Co} / \mathrm{Pd}$ multilayered film, perpendicular magnetic recording media, intergranular exchange coupling, substrate temperature, $\mathrm{N}_{2}$ gas addition

\section{Introduction}

$\mathrm{Co} / \mathrm{Pd}$ multilayered films are promising candidates for perpendicular magnetic recording media with high thermal stability because of their high perpendicular magnetic anisotropy ${ }^{1), 2)}$. However, the high medium noise of $\mathrm{Co} / \mathrm{Pd}$ multilayered media is a critical issue. The high medium noise results from large magnetic clusters brought about by strong intergranular exchange coupling along the film plane. Decreasing the intergranular exchange coupling is essential for the reduction of medium noise. Many studies have been carried out in order to decrease the intergranular exchange coupling. Appropriate sputtering conditions, e.g., deposition under high Ar gas pressure ${ }^{3)}$, sputtering using $\mathrm{Kr}$ gas instead of $\mathrm{Ar}$ gas ${ }^{4)}$, and $\mathrm{O}_{2}$ gas addition to Ar sputtering gas for deposition of $\mathrm{Co}-\mathrm{B} / \mathrm{Pd}-\mathrm{B}$ multilayered films ${ }^{5)}$, promoted isolation of the $\mathrm{Co} / \mathrm{Pd}$ crystal grains and thereby reduced the intergranular exchange coupling. In most studies, $\mathrm{Co} / \mathrm{Pd}$ multilayered films are deposited by sputtering at room temperature. Interestingly, $\mathrm{Co} / \mathrm{Pt}$ multilayered films fabricated by electron beam evaporation have been reported to show weak intergranular exchange coupling when deposited onto a heated substrate ${ }^{6)}$. The addition of $\mathrm{N}_{2}$ to $\mathrm{Ar}$ sputtering gas has also been known to result in fine crystal grain structure ${ }^{7)}$.

In this study, heating of the substrate during deposition and addition of $\mathrm{N}_{2}$ to $\mathrm{Ar}$ gas were applied to sputter-deposited $\mathrm{Co} / \mathrm{Pd}$ multilayered films. Their effects on magnetic properties, crystalline microstructure, and read-write $(R / W)$ characteristics of the Co/Pd multilayered perpendicular magnetic recording media were investigated in detail.

\section{Experiment}

The samples composed of [Co $(0.2 \mathrm{~nm}) / \mathrm{Pd}(0.8$ $\mathrm{nm})]_{20} / \mathrm{Pd}(5 \mathrm{~nm}) /$ substrate were prepared by dc magnetron sputtering. The crystallized glass disks with 2.5 inch in diameter were used as substrates. The sputtering conditions are listed in Table 1 . The substrate temperature during deposition was set to 20 or $230{ }^{\circ} \mathrm{C}$. The partial pressure of $\mathrm{N}_{2}$ gas was $0.3 \%$ of Ar gas pressure, i.e., 0.06 mTorr. The layer structure of the samples for the $R / W$ experiments was $\mathrm{C}(5 \mathrm{~nm}) /[\mathrm{Co}(0.2 \mathrm{~nm}) / \mathrm{Pd}(0.8 \mathrm{~nm})]_{20} / \mathrm{Pd}$ $(5 \mathrm{~nm}) / \mathrm{C}(5 \mathrm{~nm}) / \mathrm{CoZrNb}(300 \mathrm{~nm}) /$ substrate. The values of saturation magnetic flux density and coercivity for the CoZrNb soft magnetic underlayer (SUL) were $14 \mathrm{kG}$ and 10 Oe, respectively.

Magnetic properties were measured by using a vibrating sample magnetometer (VSM, maximum applied field $H_{\max }$ $=15 \mathrm{kOe})$, a torque magnetometer $\left(H_{\max }=20 \mathrm{kOe}\right)$, and a polar Kerr effect magnetometer $\left(H_{\max }=20 \mathrm{kOe}\right)$. Magnetic anisotropy field $\left(H_{k}\right)$ was calculated by using the following equation involving magnetic anisotropy constant $\left(K_{u}\right)$ and saturation mgnetization $\left(M_{s}\right)$ :

$$
H_{k}=2 K_{u} / M_{s} \text {. }
$$

The value of $K_{u}$ was obtained by adding a demagnetization field energy, $2 \pi M_{s}^{2}$, to the experimental value of the magnetic anisotropy constant, $K_{\text {eff. }}$. Surface roughness and magnetic domain structure of the samples were observed by an atomic force microscope (AFM) and a magnetic force microscope (MFM), respectively. The crystalline microstructure was analyzed with an X-ray diffractometer

Table 1 Sputtering conditions.

\begin{tabular}{ll|l}
\hline \multicolumn{2}{l|}{ Background pressure [Torr] } & $<3.0 \times 10^{-7}$ \\
\hline Ar pressure [mTorr] & $\mathrm{Co} / \mathrm{Pd}$ & $20\left(+\mathrm{N}_{2} 0.06\right)$ \\
& $\mathrm{Pd}$ & 100 \\
& $\mathrm{C}$ & 50 \\
& $\mathrm{CoZrNb}$ & 5 \\
\hline Substrate temperature $\left[{ }^{\circ} \mathrm{C}\right]$ & $\mathrm{Co} / \mathrm{Pd}$ & 20 or 230 \\
& $\mathrm{Pd}$ & 20 or 230 \\
& $\mathrm{C}$ & 20 \\
& $\mathrm{CoZrNb}$ & 20 \\
\hline
\end{tabular}


Table 2 Magnetic properties of $\mathrm{Co} / \mathrm{Pd}$ multilayered films.

\begin{tabular}{c|cc|ccccc}
\hline Film & Sub. temp. $\left[{ }^{\circ} \mathrm{C}\right]$ & $\mathrm{N}_{2}$ pressure $[\mathrm{mTorr}]$ & $M_{s}\left[\mathrm{emu} / \mathrm{cm}^{3}\right]$ & $H_{c}[\mathrm{kOe}]$ & $\alpha[-]$ & $D_{\text {cluster }}[\mathrm{nm}]$ & $H_{k}[\mathrm{kOe}]$ \\
\hline A & 20 & 0 & 320 & 5.3 & 3.6 & $169 \pm 30$ & 23.4 \\
B & 230 & 0 & 340 & 4.7 & 3.6 & $167 \pm 37$ & 20.0 \\
C & 20 & 0.06 & 240 & 4.2 & 2.6 & $143 \pm 25$ & 21.2 \\
D & 230 & 0.06 & 310 & 5.2 & 2.0 & $135 \pm 26$ & 17.7 \\
\hline
\end{tabular}

(XRD) using $\mathrm{Cu}-\mathrm{K} \alpha$ radiation at an acceleration voltage of $50 \mathrm{kV}$ and an emission current of $200 \mathrm{~mA}$, and with a transmission electron microscope (TEM) with an acceleration voltage of $200 \mathrm{kV}$. The compositions of the grains and grain boundaries were determined by energy dispersive X-ray spectroscopy (EDS). The $R / W$ characteristics were evaluated with a merged MR head for recording (gap length $G_{r}=0.28 \mu \mathrm{m}$, track width $T_{w}=1.45$ $\mu \mathrm{m}$ ) and reading (shield-to-shield gap length $G_{s-s}=0.2 \mu \mathrm{m}$, $\left.T_{w}=0.9 \mu \mathrm{m}\right)$. The linear velocity was $6.35 \mathrm{~m} / \mathrm{s}$. The reproduced voltage was measured as the peak-to-peak amplitude of the fundamental component of the signal by using a spectrum analyzer. The medium noise was calculated by subtracting the system noise power from the total noise power and integrating up to $50 \mathrm{MHz}$.

\section{Results and Discussion}

\subsection{Magnetic properties}

Table 2 shows magnetic properties for the $\mathrm{Co} / \mathrm{Pd}$ multilayered films used in this study. These films were deposited under four sputtering conditions (A-D); varying the substrate temperature and the $\mathrm{N}_{2}$ partial pressure (Table 2). The values of $M_{s}$, coercivity in the direction perpendicular to the film surface $\left(H_{c}\right)$, and the $M-H$ loop slope parameter $(\alpha)$ defined as $4 \pi(\mathrm{d} M / \mathrm{d} H)_{H=H c}$ were measured by using a VSM. The value of $\alpha$ is regarded as a parameter reflecting the magnitude of intergranular exchange coupling ${ }^{8)}$ and have been reported to be closely related to magnetic cluster diameter $\left(D_{\text {cluster }}\right){ }^{9)}$. The squareness ratio $\left(S Q R=M_{r} / M_{s}\right.$, where $M_{r}$ is remanent magnetization) was equal to unity within the experimental error and a nucleation field $\left(H_{n}\right)$ exhibited a large negative value $(<-2.3 \mathrm{kOe})$ for all samples used. The values of $H_{c}$
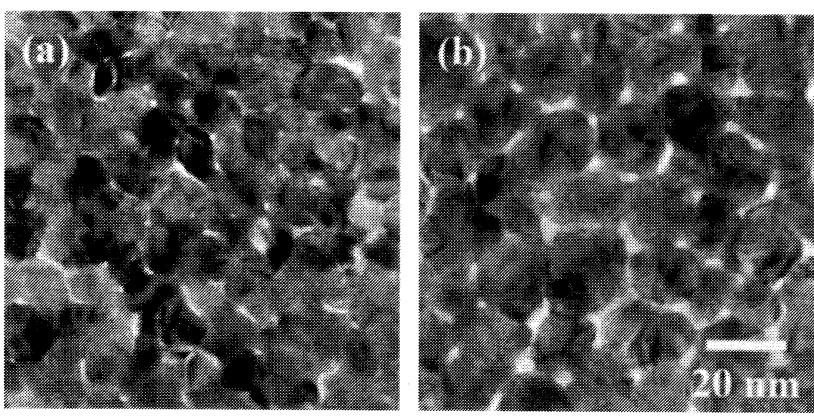

Fig. 1 Plan-view TEM bright field images of $\mathrm{Co} / \mathrm{Pd}$ multilayer for films A (a) and D (b) listed in Table 2. for films B and C are small as compared with films A and $D$. The $\alpha$ value of film $C$ is smaller than that of film $A$, which is thought to come from the decrease in not only the intergranular exchange coupling but also the exchange coupling within the grains, because the value of $M_{s}$ for film C was markedly reduced. Film D has values of $M_{s}$ and $H_{c}$ similar to film A, although it has a smaller $\alpha$ value than film A. These results reveal that intergranular exchange coupling in $\mathrm{Co} / \mathrm{Pd}$ multilayered films can be suppressed without a decrease in $H_{c}$ by depositing the films on heated substrates in an $\mathrm{Ar}+\mathrm{N}_{2}$ atmosphere.

Table 2 also shows the values of $D_{\text {cluster }}$ for films A-D. Here, they are calculated as an average and a standard deviation of diameter of circles whose areas are equal to those of magnetic domains observed in the MFM images at the ac demagnetized state. The values of $D_{\text {cluster }}$ correlate with the $\alpha$ values. Namely, the smaller magnetic clusters are obtained for films exhibiting the lower $\alpha$ values. For film $\mathrm{D}$, the decrease in intergranular exchange coupling leads to the formation of fine magnetic clusters.

\subsection{Crystalline microstructure}

Figures 1 (a) and (b) show plan-view TEM bright field images of $\mathrm{Co} / \mathrm{Pd}$ multilayer for films $\mathrm{A}$ and $\mathrm{D}$, respectively. The average and the standard deviation of the $\mathrm{Co} / \mathrm{Pd}$ crystal grain diameter $\left(D_{\text {grain }}\right)$ for these two films are $13.9 \pm 2.5$ and $19.8 \pm 3.5 \mathrm{~nm}$, respectively. The crystal grains for film D are larger than that for film A, while the grain boundaries for film D are more well-defined than those for film A. The grain boundaries turned out to be voids from EDS measurement results, consistent with the decrease in intergranular exchange coupling for film D. Nitrogen was detccted in both the grains and the grain boundaries for film D with EDS, but the two-dimensional distribution of nitrogen composition could not be determined. The formation of nitrides was not confirmed in the electron diffraction patterns.

Figure 2 (a) shows the XRD patterns of films A-D together with that of the glass substrate. Figure 2 (b) represents XRD patterns where the glass substrate contribution is subtracted and only the reflection peak from fcc-Co/Pd(111) is observed. The $2 \theta$ values for the fcc-Co/Pd(111) of all the films varies within $\pm 0.1 \mathrm{deg}$., but in this study these differences are considered to be due to a systematic error in determining the origin of the $2 \theta$ angle. Film $\mathrm{C}$ deposited with $\mathrm{N}_{2}$ additive gas shows a smaller peak than film A without it. In the case of films deposited onto a heated substrate, the peak intensity of film $\mathrm{D}$ deposited with $\mathrm{N}_{2}$ additive gas is similarly lower 

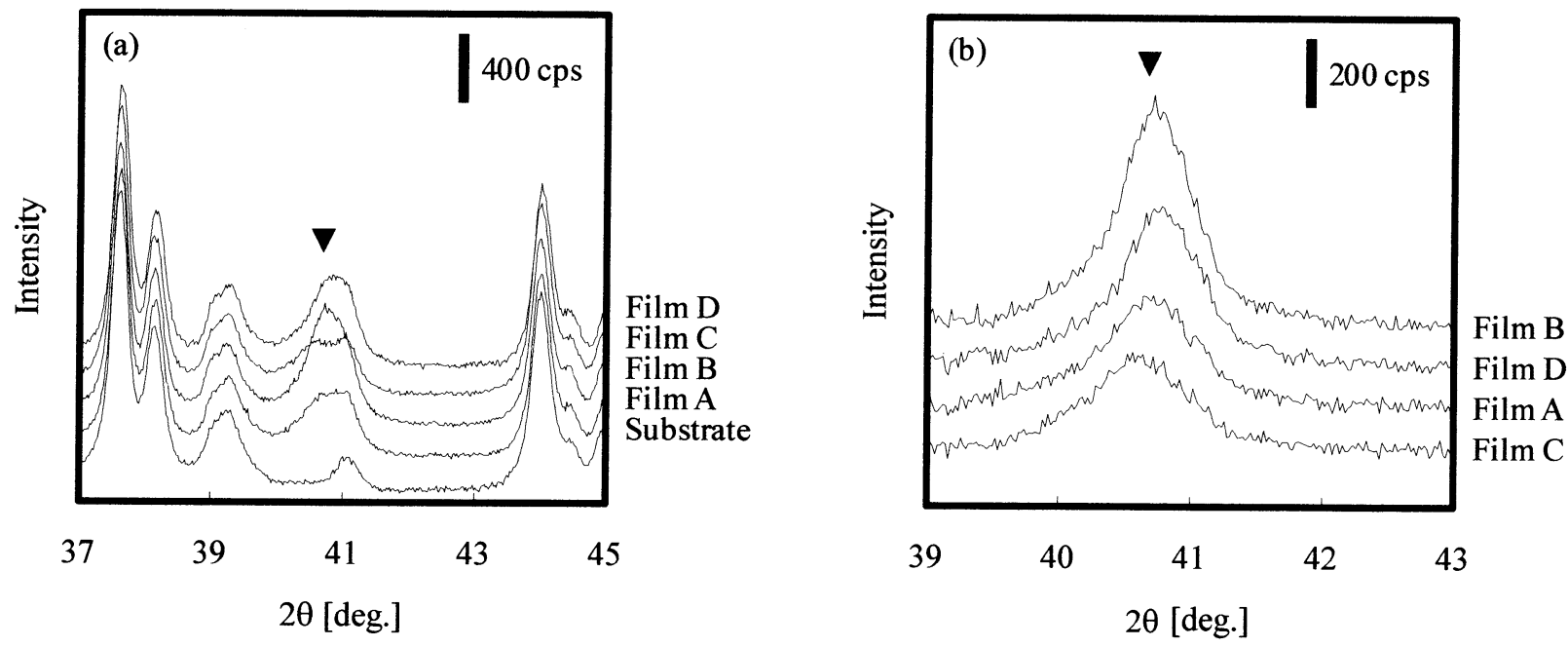

Fig. 2 (a) XRD patterns in the range of $2 \theta=37-45$ deg. of films A, B, C, and D together with that of glass substrate. (b) XRD patterns in the range of $2 \theta=39-43$ deg., where the counts of glass substrate are subtracted. The sign $\nabla$ indicates the peak for fcc-Co/Pd (111) reflection.

than that of film B without it. The decrease in the peak intensity is thought to be attributed to the suppression of the growth of $\mathrm{Co} / \mathrm{Pd}$ crystal grains by adding $\mathrm{N}_{2}$. The peak intensities of films B and D deposited on the heated substrate are greater than those of films $\mathrm{A}$ and $\mathrm{C}$ deposited at room temperature, respectively. This result indicates that the deposition on the heated substrates enlarges the $\mathrm{Co} / \mathrm{Pd}$ crystal grains and/or enhances the fcc-Co/Pd(111) preferred orientation. By comparing the peak intensities of fcc-Co/Pd(111) in XRD measurements and the values of $D_{\text {grain }}$ obtained from TEM observation between films A and $\mathrm{D}$, we infer that the increase in the peak intensity reflects the enlargement of the $\mathrm{Co} / \mathrm{Pd}$ crystal grains. The heating of the substrate during deposition enhances the $\mathrm{Co} / \mathrm{Pd}$ grains growth in the lateral direction, which was obscrved as in the casc of the Co/Pt films deposited with electron beam evaporation ${ }^{6)}$. Film B deposited on the

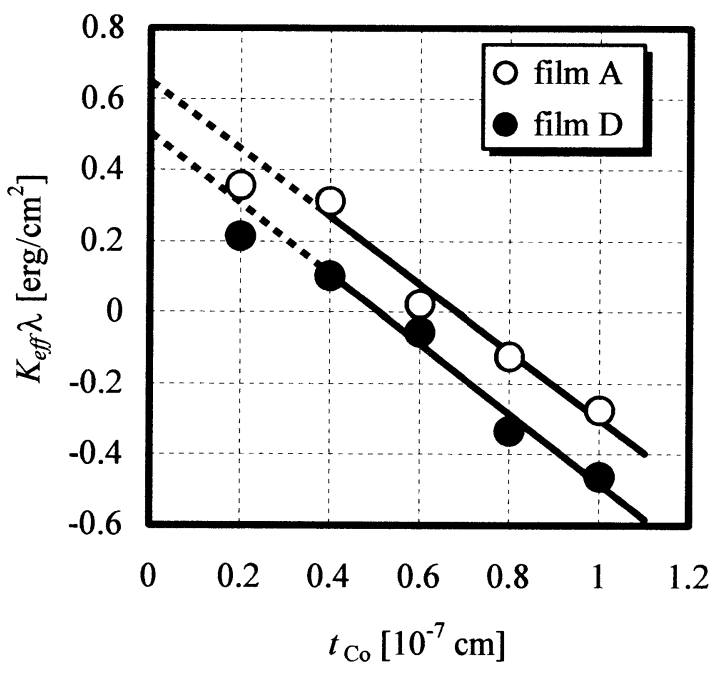

Fig. 3 Relationship between $K_{\text {eff }} \lambda$ and $t_{\mathrm{Co}}$. heated substrate is considered to consist of larger grains than film $\mathrm{A}$, although the values of $D_{\text {cluster }}$ for both films are similar. This suggests that the isolation of $\mathrm{Co} / \mathrm{Pd}$ grains is promoted and the clustering of magnetic domains is suppressed by raising the substrate temperature during deposition as reported in the previous paper ${ }^{6)}$. Especially in this study, the effect of deposition on high substrate temperatures is found to be pronounced in the case of film D deposited with $\mathrm{N}_{2}$ additive gas.

\subsection{Evaluation of surface magnetic anisotropy}

Film D exhibits weaker intergranular exchange coupling than film A, but at the same time it has a slightly lower perpendicular magnetic anisotropy, as shown in Table 2. The origin of perpendicular magnetic anisotropy in $\mathrm{Co} / \mathrm{Pd}$ multilayered films have been said to be surface magnetic anisotropy at the interfaces of $\mathrm{Co}$ and Pd layers ${ }^{1)}$. We evaluated the surface magnetic anisotropy of films A and D from the relationship between $K_{\text {eff }} \lambda$ and $t_{\text {Co }}$, as shown in Fig. 3 , by using the following phenomenological equation 10).

$$
K_{\text {eff }} \lambda=2 K_{s}+K_{v} t_{\mathrm{Co}}
$$

where $\lambda$ is the $\mathrm{Co} / \mathrm{Pd}$ bi-layer period, $K_{s}$ is surface magnetic anisotropy energy, $K_{v}$ is volume magnetic anisotropy energy, and $t_{\mathrm{Co}}$ is the thickness of a constituent Co layer. Table 3 lists the values of $K_{s}$ and $K_{v}$ for films A and $\mathrm{D}$, which are determined as the intercept with the ordinate and as the slope of the straight line obtained by the least-square fitting in Fig. 3, respectively. There is little difference between the $K_{v}$ values of these two films,

Table 3 Values of $K_{s}$ and $K_{v}$ for films A and D.

\begin{tabular}{c|cc}
\hline Film & $K_{s}\left[\mathrm{erg} / \mathrm{cm}^{2}\right]$ & $K_{v}\left[10^{6} \mathrm{erg} / \mathrm{cm}^{3}\right]$ \\
\hline A & 0.325 & -9.52 \\
D & 0.252 & -9.89 \\
\hline
\end{tabular}




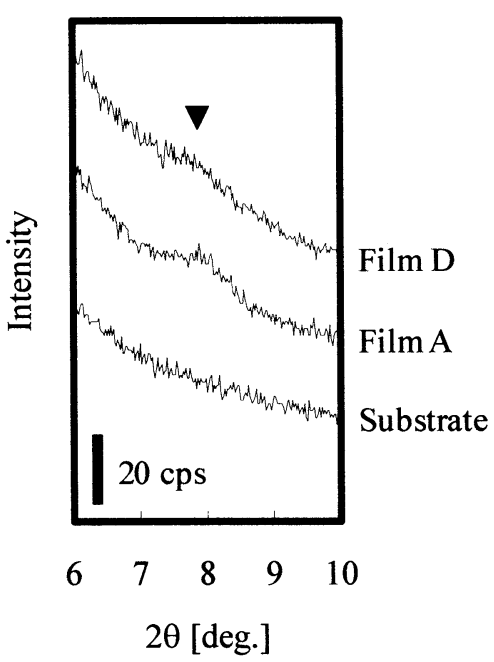

Fig. 4 XRD patterns in the range of $2 \theta=6-10$ deg. of films A and $\mathrm{D}$ together with that of glass substrate. The sign $\boldsymbol{\nabla}$ indicates the peak corresponding to the bi-layer periodicity of Co and Pd layers.

but the value of $K_{s}$ for film D is smaller than that for film A. The degradation of perpendicular magnetic anisotropy in film D is caused by the decrease in surface magnetic anisotropy energy.

An XRD measurement in the low angle region of $2 \theta$ enables us to evaluate the regularity of periodic bi-layer structure of $\mathrm{Co}$ and Pd layers. Figure 4 shows XRD patterns in the range of 6 to $10 \mathrm{deg}$. of films $A$ and D together with the result of the glass substrate. The peak intensity in the low angle region corresponding to the regularity of bi-layer structure for film $\mathrm{D}$ is lower than that for film A, whereas film D has larger Co/Pd crystal grains and exhibits a greater peak intensity for fcc-Co/Pd(111) than film A as shown in Figs. 1 and 2, respectively. These results indicate the deterioration of the regularity of the bi-layer structure in film $D$. This could be due to inter-diffusion between Co and Pd sublayers, which was promoted by heating the substrate during deposition. From AFM observations, deposition on the heated substrate and with $\mathrm{N}_{2}$ additive gas increased surface roughness of the films, which may also lead to the bi-layer structure deterioration. The irregularity in the bi-layer structure decreased the surface magnetic anisotropy energy, causing a low perpendicular magnetic anisotropy.

\subsection{Application to double-layered media}

It was confirmed that the sputtering process proposed in this study was useful for the fabrication of $\mathrm{Co} / \mathrm{Pd}$ multilayered films with weak intergranular exchange coupling $(\alpha \sim 2)$. The above deposition process was applied to the recording layer of double-layered media with a SUL. Magnetic properties and $R / W$ characteristics for the media using films $A$ and $D$ as the recording layer, which were designated as media A' and D', respectively, are listed in Table 4. Magnetic properties for the media were measured by using a Kerr effect magnetometer. For estimation of $\alpha$ values, values of $M_{s}$ for single-layered films measured with the VSM were used. The tendencies of the magnetic properties are not affected by the addition of an SUL.

The reproduced voltage for medium D' was similar to that for medium $\mathrm{A}^{\prime}$. On the other hand, medium $\mathrm{D}^{\prime}$ exhibited much lower medium noise than medium $A^{\prime}$, especially at high linear recording density over $200 \mathrm{kFRPI}$. As a result, $S / N$ ratio for medium D' is higher by 7-8 dB than that for medium $\mathrm{A}^{\prime}$ at over $200 \mathrm{kFRPI}$, as shown in Table 4. It is considered that the decreases in intergranular exchange coupling and magnetic cluster diameter lead to the improvement of $R / W$ characteristics in medium D'. The perpendicular magnetic anisotropy for film $D$ is slightly less than that for film A, as shown in Table 2 and Fig. 3. However, the reproduced voltage at the low linear recording density of $2 \mathrm{kFRPI}$ for medium $\mathrm{D}^{\prime}$ remained up to 1800 seconds analogous to medium $\mathrm{A}^{\prime}$, owing to the $S Q R$ of unity and a large value of $-H_{n}$.

\section{Conclusion}

The effects of substrate temperature and $\mathrm{N}_{2}$ gas addition to Ar sputtering gas on magnetic properties, especially intergranular exchange coupling, and crystalline microstructure of $\mathrm{Co} / \mathrm{Pd}$ multilayered films were investigated. By depositing $\mathrm{Co} / \mathrm{Pd}$ multilayered films at $230{ }^{\circ} \mathrm{C}$ with $\mathrm{N}_{2}$ partial pressure of $0.06 \mathrm{mTorr}$, the intergranular exchange coupling was suppressed without a decrease in $H_{c}$, which came from the enhancement in the physical isolation of $\mathrm{Co} / \mathrm{Pd}$ crystal grains. This film possessed smaller magnetic clusters and exhibited higher $S / N$ ratio than the film deposited at room temperature without $\mathrm{N}_{2}$ gas addition. These results indicate that the sputtering process proposed in this study was effective for fabricating low noise $\mathrm{Co} / \mathrm{Pd}$ multilayered double-layered media.

\section{Acknowledgement}

This work was carried out at the "Center for Practical Nano-Chemistry" for the 21C-COE Programme of the Ministry of Education, Culture, Sports, Science and Technology (MEXT), Japan, and was also financially

Table 4 Magnetic properties and $R / W$ characteristics for double-layered media.

\begin{tabular}{c|cc|ccc}
\hline Medium & Sub. temp. $\left[{ }^{\circ} \mathrm{C}\right]$ & $\mathrm{N}_{2}$ pressure [mTorr] & $H_{c}[\mathrm{kOe}]$ & $\alpha[-]$ & $S_{p-p} / N_{\text {rms }}$ ratio (at 200 kFRPI) \\
\hline $\mathrm{A}^{\prime}$ & 20 & 0 & 6.1 & 5.3 & 0.7 \\
$\mathrm{D}^{\prime}$ & 230 & 0.06 & 5.7 & 1.9 & 8.2 \\
\hline
\end{tabular}


supported by Center of Excellence Research on "Establishment of Molecular Nano-Engineering by Utilizing Nanostructure Arrays and its Development into Micro-System" from MEXT. The authors express their gratitude to Dr. K. Tagami and Mr. N. Ohshima, NEC Corporation, for their cooperation in the torque measurement.

\section{References}

1) P. F. Carcia, A. D. Meinhaldt, A. Suna: Appl. Phys. Lett., 47, 178 (1985).

2) B. M. Lairson, J. Perez, C. Baldwin: Appl. Phys. Lett., 64, 2891 (1994).

3) L. Wu, S. Yanase, N. Honda, K Ouchi: IEEE Trans. Magn., 33, 3094 (1997).

4) W. Peng, O. Keitel, R. H. Victora, E. Koparal, J. H. Judy: IEEE Trans. Magn., 36, 2390 (2000).
5) H. Ohmori, A. Maesaka: J. Magn. Magn. Mater., 235, 45 (2001).

6) D. Weller, L. Folks, M. Best, E. E. Fullerton, B. D. Terris, G. J. Kusinski, K. M. Krishnan, G. Thomas: J. Appl. Phys., 89, 7525 (2001).

7) Y. Sano, H. Muraoka, I. Watanabe, Y. Nakamura: J. Magn. Magn. Mater., 155, 212 (1996).

8) I. Tagawa, A. Takeo, Y. Nakamura: J. Magn. Magn. Mater., 155, 341 (1996).

9) O. Kitakami, Y. Shimada: Jpn. J. Appl. Phys., 40, 4019 (2001).

10) H. J. G. Draaisma, W. J. M. de Jonge, F. J. A. den Broeder: J. Magn. Magn. Mater., 66, 351 (1987).

Received October 9, 2002; Accepted January 17, 2003. 